\title{
Weak Localization and Mobility in ZnO Nanostructures
}

\section{Citation}

Likovich, Edward M., Kasey J. Russell, Eric W. Petersen, and Venkatesh Narayanamurti. 2009. Weak localization and mobility in ZnO nanostructures. Physical Review B 80(24): 245318.

\section{Published Version}

doi:10.1103/PhysRevB.80.245318

\section{Permanent link}

http://nrs.harvard.edu/urn-3:HUL.InstRepos:3606239

\section{Terms of Use}

This article was downloaded from Harvard University's DASH repository, and is made available under the terms and conditions applicable to Open Access Policy Articles, as set forth at http:// nrs.harvard.edu/urn-3:HUL.InstRepos:dash.current.terms-of-use\#OAP

\section{Share Your Story}

The Harvard community has made this article openly available.

Please share how this access benefits you. Submit a story.

Accessibility 


\title{
Weak localization and mobility in $\mathrm{ZnO}$ nanostructures
}

\author{
Edward M. Likovich, ${ }^{*}$ Kasey J. Russell, Eric W. Petersen, and Venkatesh Narayanamurti \\ School of Engineering and Applied Sciences, \\ Harvard University, Cambridge, Massachusetts 02138
}

(Dated: December 22, 2009)

\begin{abstract}
We conduct a comprehensive investigation into the electronic and magneto-transport properties of $\mathrm{ZnO}$ nanoplates grown concurrently with $\mathrm{ZnO}$ nanowires by the vapor-liquid-solid (VLS) method. We present magnetoresistance data showing weak localization in our nanoplates and probe its dependence on temperature and carrier concentration. We measure phase coherence lengths of 50-100 nm at $1.9 \mathrm{~K}$, and, because we do not observe spin-orbit scattering through antilocalization, suggest that $\mathrm{ZnO}$ nanostructures may be promising for further spintronic study. We then proceed to study the effect of weak localization on electron mobility using 4-terminal van der Pauw resistivity and Hall measurements versus temperature and carrier concentration. We report an electron mobility of $\sim 100 \frac{\mathrm{cm}^{2}}{V s}$ at $275 \mathrm{~K}$, comparable to what is observed in ZnO thin films. We compare Hall mobility to field-effect mobility, which is more commonly reported in studies on $\mathrm{ZnO}$ nanowires, and find that field-effect mobility tends to overestimate Hall mobility by a factor of 2 in our devices. Finally, we comment on temperature-dependent hysteresis observed during transconductance measurements and its relationship to mobile, positively-charged Zn interstitial impurities.

PACS numbers:
\end{abstract}




\section{INTRODUCTION}

$\mathrm{ZnO}$ has been extensively studied due to its favorable optoelectronic properties which include a wide bandgap (3.37 eV), strong exciton binding energy (60 meV), and potential use as a transparent conductor in light-emitting and -collecting devices. ${ }^{1-3}$ P-type doping of $\mathrm{ZnO}$ has recently been demonstrated in both bulk films ${ }^{4,5}$ and nanowires, ${ }^{6}$ opening the door for a variety of devices based on p-n junctions. Additionally, ZnO nanowires have shown promise in applications that include gas sensors, which take advantage of their high surface area to volume ratio, ${ }^{7}$ and room-temperature spintronics, as the incorporation of transition metals has been shown to induce ferromagnetism in both bulk $\mathrm{ZnO}^{8}$ and $\mathrm{ZnO}$ nanowires. ${ }^{9,10} \mathrm{ZnO}$ nanowires have also demonstrated amplified spontaneous emission ${ }^{11}$ and room temperature UV lasing due to their high exciton binding energy. ${ }^{12}$ For a review of $\mathrm{ZnO}$ device applications, see Ref. 13.

Much recent research has focused on spin injection and transport in semiconductors, potentially leading the way to a new paradigm of electronics based on spin. ${ }^{14-16}$ The interest of spin-dependent transport has extended to $\mathrm{ZnO}$ thin films doped with $\mathrm{Mn}$, which have shown interesting spin-orbit and s-d exchange interactions. ${ }^{17}$ However, there has not been a significant investigation of magneto-transport in $\mathrm{ZnO}$ nanostructures, despite a wide interest in nanostructure devices.

In this paper we report on the electronic and magneto-transport properties of $\mathrm{ZnO}$ nanoplates, structures as thin as typical ZnO nanowires ( 100 nm) but several microns long and wide, that are grown simultaneously alongside nanowires by the vapor-liquid-solid (VLS) method. The nanoplate geometry, in contrast to that of a nanowire, allows us to make 4-wire Hall and van der Pauw resistivity measurements. We believe our results generally apply to $\mathrm{ZnO}$ nanowires as well, since they are also expected to exhibit three-dimensional electron transport properties, as the diameters of typical ZnO nanowires (30-100 nm) are insufficiently small to establish quantum confinement in the radial direction. ${ }^{18}$

We present data that represent the first observation of weak localization in $\mathrm{ZnO}$ nanostructures and probe its dependence on temperature and carrier concentration. From this we are able to determine the inelastic scattering lifetime of electrons, and, due to an absence of anti-localization, believe that the spin-orbit scattering lifetime is significantly longer than the inelastic scattering lifetime. We then consider how weak localization affects mobility and 
carrier transport by conducting Hall measurements for different temperatures and carrier concentrations. We compare these values with the 2-terminal field-effect approximation commonly reported in the literature but which suffer from parasitic contact resistance and rely on assumptions about the geometric extent of the nanowire conduction channel. ${ }^{19-22}$ Recent work suggests that there may be independent core and surface conduction mechanisms, ${ }^{23}$ which would need to be taken into consideration to accurately determine the field-effect or effective mobility. Finally, we comment on temperature-dependent hysteresis observed during transconductance measurements and its possible relationship to mobile, charged impurities.

\section{FABRICATION AND EXPERIMENTAL SETUP}

We fabricated $\mathrm{ZnO}$ nanoplates using the Vapor-Liquid-Solid (VLS) growth mechanism, first demonstrated by Wagner and Ellis. ${ }^{24}$ The growth apparatus used for this experiment consisted of a single-zone tube furnace (from Lindberg/Blue) through which we passed a metered flow of $\mathrm{Ar}$ and $\mathrm{O}_{2}$ in a 1000:1 ratio. A quartz sample holder, containing both the Si (111) substrate (with $5 \mathrm{~nm}$ diameter Au catalyst nanoparticles dispersed onto it) and a mixture of $\mathrm{ZnO}$ powder and graphite (1:1 ratio by volume), was used to move the substrate in and out of the hot zone of the furnace, thereby initiating and terminating nanowire growth. After a growth of 45 minutes at $975^{\circ} \mathrm{C}, \mathrm{ZnO}$ nanowires and nanoplates were present on the substrate. The nanoplates had gold catalyst particles at their tip (characteristic of VLS growth) and were single-crystalline, as shown in Figure 1a. Atomic force (AFM) and scanning electron microscopy (SEM) analysis indicated that the nanoplates were roughly the same thickness as typical nanowires $(\sim 100 \mathrm{~nm})$ but extended several microns laterally, as shown in Figure 1b. The growth of similar nanoplates has been reported in the literature for both VLS ${ }^{25}$ and solution synthesis ${ }^{26}$ methods. We offer the additional observation that $\mathrm{ZnO}$ nanoplates tend to preferentially grow on substrates that form a native oxide layer; we observed them on both Si (111) and Ti foil substrates, but not on Mo foil. ${ }^{27}$

To experimentally probe the nanoplates, we first removed them from the substrate by sonication in isopropanol for short bursts of several seconds. This created an isopropanol solution containing suspended nanoplates. We then used a pipet to deposit droplets of the solution onto a p++ Si wafer with a $300 \mathrm{~nm}$ thermally grown oxide layer. We allowed the droplet to air dry, recorded the positions of the deposited nanostructures, and used 

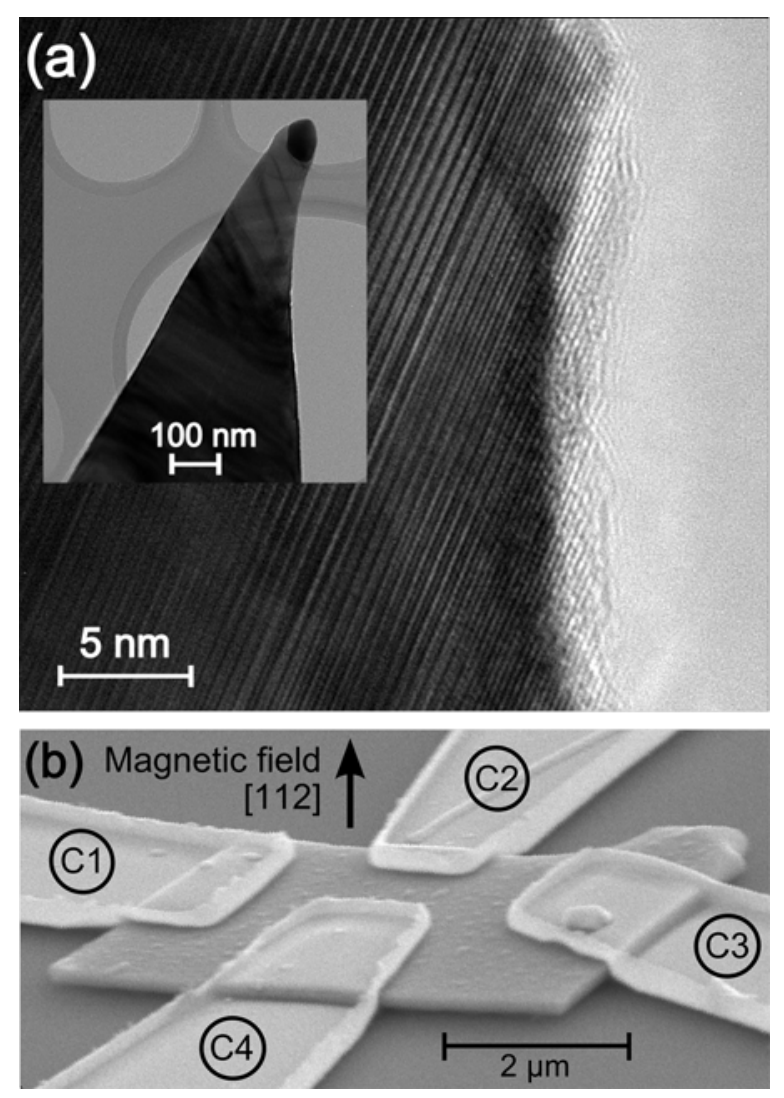

FIG. 1: (Color online) (a) Transmission electron microscopy (TEM) atomic-resolution image showing the crystalline nature of our nanoplates. (Inset) TEM image taken at lower magnification showing the Au catalyst at the end of the nanoplate, which is characteristic of vapor-liquid-solid growth. (b) Scanning electron microscopy image of a $\mathrm{ZnO}$ nanoplate device. The four contacts are labeled $\mathrm{C} 1, \mathrm{C} 2, \mathrm{C} 3$, and $\mathrm{C} 4$. A $300 \mathrm{~nm} \mathrm{SiO}_{2}$ layer serves as the gate barrier with the gate contact to the backside of the heavily-doped Si substrate.

electron-beam lithography to define 4 contacts (sputtered $20 \mathrm{~nm} \mathrm{Ti}$, followed by $80 \mathrm{~nm} \mathrm{Au}$ ) to each nanoplate, as shown in Figure 1b. These contact metals resulted in ohmic behavior at the biases measured and are commonly used in the literature. ${ }^{28,29}$ An In back contact was cold-pressed to the Si substrate to control carrier concentration through gating. The devices were then placed in a variable temperature cryostat capable of applying a magnetic field perpendicular to the surface of the device, which was identified as the [112] direction by transmission electron microscopy (TEM) diffraction patterns. A total of 8 devices were measured, all of which exhibited similar behavior. 


\section{RESISTIVITY}

As the devices were cooled, we measured resistivity as a function of temperature using the van der Pauw formulation, which enables the resistivity of a planar sample of arbitrary shape to be determined with corrections for contact anisotropy. ${ }^{30,31}$ To do so, we sourced a sinusoidal A.C. current of $30 \mathrm{nA}$ RMS between contacts C1 and C2 (see Figure 1) and concurrently measured voltage between contacts $\mathrm{C} 3$ and $\mathrm{C} 4$ with a lock-in amplifier. Immediately following this, contacts $\mathrm{C} 1$ and $\mathrm{C} 4$ were swapped and a second measurement was taken such that current was sourced between contacts C2 and C3 and voltage was concurrently measured between contacts $\mathrm{C} 4$ and $\mathrm{C} 1$.

As shown in Figure 2, resistivity increases linearly with T at high temperatures (200$300 \mathrm{~K}$ ), as expected for phonon-dominated scattering. For low temperatures (below 150 $\mathrm{K})$, resistivity scales exponentially in $1 / \mathrm{T}$, characteristic of a lightly-doped semiconductor. ${ }^{32}$ We observed a minimum resistivity around $180 \mathrm{~K}$, which has been reported in the $\mathrm{ZnO}$ literature and likely represents the point at which the additive resistive contributions of phonon scattering and electron weak localization are at a minimum. ${ }^{33,34} \mathrm{~A}$ discussion of electron weak localization, which opposes diffusive transport at low temperatures, is covered extensively in the next section.

We observed a distinct activation energy characterized by the linear region in the semi-log plot of resistivity shown in Figure 2. The activation energy was obtained by fitting the data to an exponential functional form in the limit of no compensation, $\exp \left(-E_{a} / 2 k T\right)$, where $E_{a}$ is the activation energy; $k$ is the Boltzmann constant; and $T$ is the temperature. From this analysis, we calculate $E_{a}=32 \mathrm{meV}$. This $32 \mathrm{meV}$ activation energy is consistent with reports in the literature for Zn interstitials, which act as electron donors. ${ }^{35}$

\section{WEAK LOCALIZATION}

Electron weak localization originates from the constructive interference of backscattered electronic wavefunctions which increases the probability of localizing an electron. This phenomenon manifests itself by a positive correction to resistivity at zero magnetic field, which is easily broken by applying a magnetic field to destroy coherent superposition. For a more thorough review of weak localization, see Refs. 36,37. 


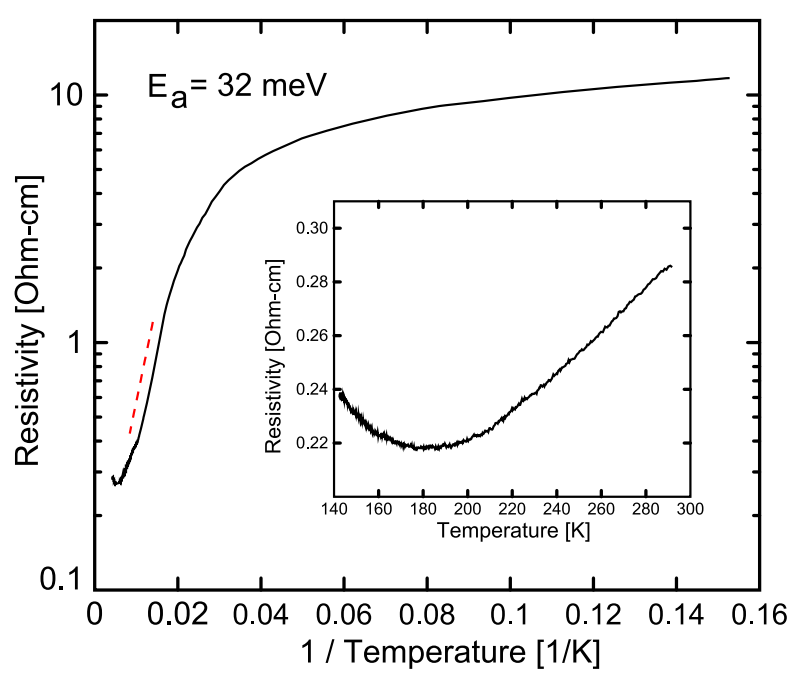

FIG. 2: (Color online) Semi-log plot of van der Pauw resistivity versus 1/T. The resistivity exhibits an exponential dependence in $1 / \mathrm{T}$, and we observe a distinct activation energy of $32 \mathrm{meV}$ that corresponds to $\mathrm{Zn}$ interstitials. The red, dashed line is meant to guide the eye to the linear region from which activation energy is extracted. (Inset) High temperature van der Pauw resistivity showing an absolute minimum near $180 \mathrm{~K}$, above which resistivity scales linearly with $\mathrm{T}$, characteristic of electron-phonon scattering.

We observed weak localization as a positive correction to van der Pauw resistivity at zero magnetic field for a variety of carrier concentrations and temperatures as presented in Figure 3 (see Figure 1b for experimental setup). As expected, the effect was temperaturedependent and was strongest at low temperatures (10 K and below), with a very small effect at $16 \mathrm{~K}$ and no effect at $33 \mathrm{~K}$. The effect dies out for higher temperatures because the inelastic scattering lifetime, $\tau$, becomes comparable to the elastic scattering lifetime, at which point the phase coherence necessary for weak localization is destroyed. We also found the effect to be dependent on carrier concentration, with higher carrier concentrations yielding larger corrections to resistivity. We attribute this to the larger fractional change in resistivity, $\Delta \rho / \rho$, caused by the reduction in absolute resistivity, $\rho$, accompanying higher carrier concentrations. The observed increase in $\tau$ with carrier density agrees with reports of weak localization in Si MOSFETs. ${ }^{38}$

The observation of weak localization has been reported in the literature for $\mathrm{ZnO}$ thin films doped with $\mathrm{Al},{ }^{39} \mathrm{~B},{ }^{40}$ and transition metals, ${ }^{41}$ but not yet in $\mathrm{ZnO}$ nanostructures. Contrary to Refs. 39,41, we observe no evidence of anti-localization (a negative correction 

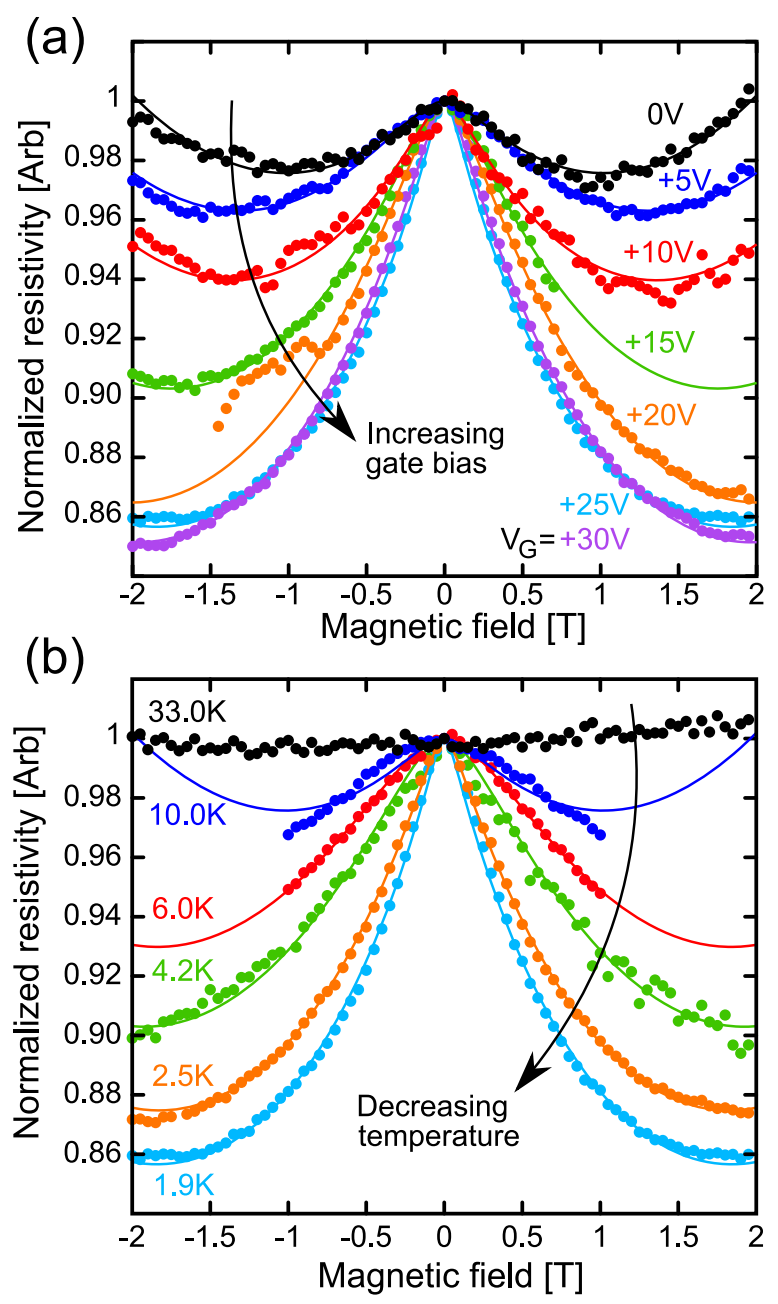

FIG. 3: (Color online) Magnetoresistance measurements of weak localization. (a) A series of gate biases (and thus carrier densities) at constant temperature $(1.9 \mathrm{~K})$. Experimental data are plotted as colored circles, with solid lines corresponding to fits to Eq. (1). The corresponding carrier concentrations range from $1.1 \times 10^{17} \mathrm{~cm}^{-3}$ for $V_{G}=0 \mathrm{~V}$ to $7.4 \times 10^{17} \mathrm{~cm}^{-3}$ for $V_{G}=+30 \mathrm{~V}$. (b) A series of temperatures at constant gate bias $\left(V_{G}=+30 \mathrm{~V}\right)$. The effect is destroyed for high temperatures due to loss of phase coherence from increased inelastic scattering.

to resistivity at zero magnetic field caused by spin-orbit scattering) for temperatures as low as $1.9 \mathrm{~K}$. This suggests that the spin-orbit scattering lifetime is significantly longer than the inelastic scattering lifetime in natively-doped $\mathrm{ZnO}$ nanostructures.

The theory behind weak localization is readily found in the literature for both twodimensional $^{42}$ and three-dimensional ${ }^{43,44}$ systems. Because of their size, we expect our nanoplates to exhibit three-dimensional transport, and as we will show, the theory of weak 
localization in three dimensions provides an excellent description of the transport that we observe. For three-dimensional systems, the correction to resistivity, $\Delta \rho / \rho$, scales as $\mathrm{B}^{2}$ for small magnetic fields and as $\mathrm{B}^{1 / 2}$ for large magnetic fields, according to:

$$
\frac{\Delta \rho}{\rho}=-\alpha \rho \frac{e^{2}}{2 \pi^{2} \hbar} \sqrt{\frac{e B}{\hbar}} F(x),
$$

where $\frac{\Delta \rho}{\rho}$ is the fractional change in resistivity due to applied magnetic field; $\alpha$ is a coefficient between 0 and 1 that is thought to represent Coulomb screening; $\rho$ is the resistivity; $e$ is the electronic charge; $\hbar$ is Planck's constant; $B$ is the applied magnetic field; and $F(x)$ is a Hurwitz zeta function given by:

$$
\begin{gathered}
F(x)=\sum_{n=0}^{\infty}\left[2(n+1+1 / x)^{1 / 2}-2(n+1 / x)^{1 / 2}\right. \\
\left.-(n+1 / 2+1 / x)^{-1 / 2}\right]
\end{gathered}
$$

where $x$ is a dimensionless quantity defined by $x=4 e D \tau B / \hbar ; D$ is the electron diffusion constant; and $\tau$ is the electron inelastic scattering lifetime. We analyzed our data in a similar manner to that of Ref. 45 and used a nonlinear least squares method to fit the data using two free parameters, $\alpha$ and $\tau$. For high magnetic fields, a positive dependence on $\mathrm{B}^{2}$ was observed and is characteristic of the usual quadratic magnetoresistance observed in bulk semiconductors. ${ }^{45}$ We accounted for this by including a temperature independent quadratic term, obtained by fitting to high-temperature data. Error bars in Figure 4 indicate the 95\% confidence intervals of the fits.

As shown in Figure 4a, calculated inelastic scattering lifetimes scale with temperature as $\mathrm{T}^{-3}$, characteristic of inelastic scattering by electron-phonon processes. ${ }^{46}$ There was only a slight observed dependence on carrier concentration (gate bias); the inelastic scattering lifetime was $5.7 \pm 0.4 \mathrm{~ns}$ at a carrier concentration of $7.4 \times 10^{17} \mathrm{~cm}^{-3}$ at $1.9 \mathrm{~K}$ compared to $3.0 \pm 0.8 \mathrm{~ns}$ at a carrier concentration of $1.3 \times 10^{17} \mathrm{~cm}^{-3}$ at $1.9 \mathrm{~K}$. Additionally, we calculate the phase coherence length, $\ell_{\phi}=\sqrt{D \tau}$, which is plotted versus temperature in Figure $4 \mathrm{~b}$. Here, we see temperature dependence that scales as $\mathrm{T}^{-3 / 4}$, the same behavior that was reported for doped $\mathrm{ZnO}$ thin films in Ref. 39. Although our material is not degenerately doped, we must still use the generalized form of the Einstein relation to analyze the temperature dependence of $D$ at the low temperatures reported in Figure $4 .{ }^{47}$ This is because the carrier density in our sample is controlled by the gate bias and does not vary with temperature in 
this temperature range. The temperature dependence of $D$ is therefore simply proportional to that of $\mu$, which should scale as $\mathrm{T}^{3 / 2}$ in the impurity-dominated regime. ${ }^{48}$ We therefore expect $\ell_{\phi} \sim \mathrm{T}^{-3 / 4}$, consistent with the observed behavior.

Measured phase coherence lengths range from $27 \pm 5 \mathrm{~nm}$ at $10 \mathrm{~K}$ to $93 \pm 13 \mathrm{~nm}$ at $1.9 \mathrm{~K}$ for $V_{G}=+30 \mathrm{~V}$. These values are roughly an order of magnitude lower than the report on heavily-doped $\mathrm{ZnO}$ thin films in Ref. 39; we believe this to be a result of their significantly larger carrier concentration $\left(10^{20} \mathrm{~cm}^{-3}\right.$ compared to $10^{17} \mathrm{~cm}^{-3}$ in our device) that effectively screens charged impurities. Our results suggest that $\mathrm{ZnO}$ may be useful for spintronic studies, given that we observe coherence lengths of 10-100 nm and no evidence of spin-orbit scattering through anti-localization.

\section{HALL MOBILITY MEASUREMENTS}

Hall mobility measurements were conducted as a function of both temperature and carrier concentration. Devices were measured in a typical Hall configuration: a sinusoidal A.C. current of $30 \mathrm{nA}$ RMS was sourced between contacts C2 and C4, and the Hall voltage was concurrently measured between contacts C1 and C3 (see Figure 1b) while sweeping the perpendicular magnetic field. Temperature was varied from $1.9 \mathrm{~K}$ to $275 \mathrm{~K}$ and carrier concentration was modulated by applying a gate bias to the $\mathrm{p}++\mathrm{Si}$ substrate relative to the current sink, C4. Gate bias was applied in $5 \mathrm{~V}$ steps from $0 \mathrm{~V}$ to $+30 \mathrm{~V}$, corresponding to carrier densities between $1.1 \times 10^{17}$ and $7.4 \times 10^{17} \mathrm{~cm}^{-3}$ at $1.9 \mathrm{~K}$ and between $8.6 \times 10^{17}$ and $1.5 \times 10^{18} \mathrm{~cm}^{-3}$ at $275 \mathrm{~K}$. We infer that our devices are n-type doped by observing an increase in conductivity for a positive applied gate bias.

Using the change in Hall voltage versus magnetic field, we calculate Hall mobility ${ }^{31}$ and plot it as a function of both temperature and carrier concentration in Figure 5. We report a Hall mobility of $103 \pm 5 \frac{\mathrm{cm}^{2}}{V s}$ for $0 \mathrm{~V}$ gate bias at $275 \mathrm{~K}$. By increasing gate bias to $+30 \mathrm{~V}$ at $275 \mathrm{~K}$, the mobility increases to $131 \frac{\mathrm{cm}^{2}}{V s}$. These values are comparable to those reported in the literature for $\mathrm{ZnO}$ thin films grown on sapphire by $\mathrm{PLD}^{49}$ and $\mathrm{MBE},{ }^{50,51}$ but are an

order of magnitude larger than those reported on $\mathrm{ZnO}$ nanowires, ${ }^{21,34}$ with the exception of nanowires with surface passivation treatments that have field-effect mobilities above 1000 $\frac{c m^{2}}{V s} \cdot 22$

As temperature decreases, electron mobility first increases due to reduced electron-phonon 

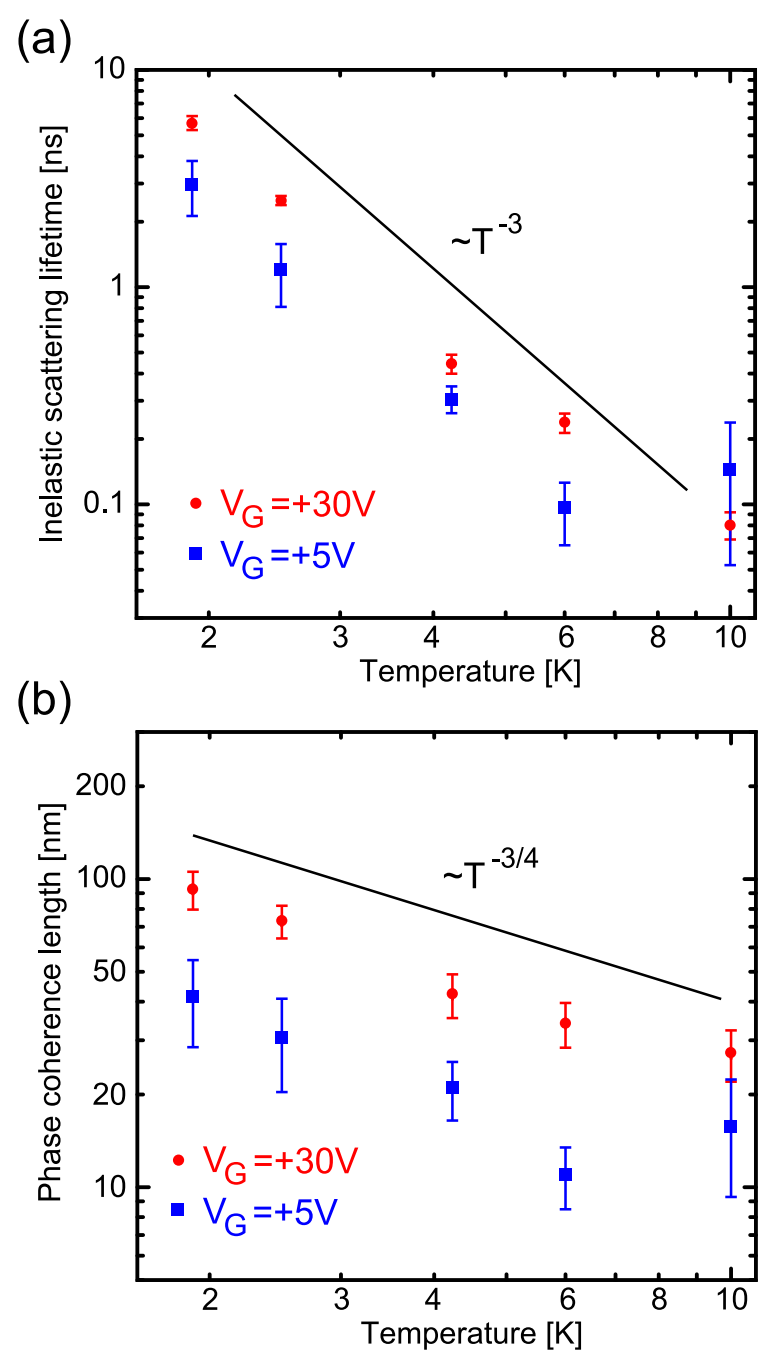

FIG. 4: (Color online) (a) Electron inelastic scattering lifetime versus temperature for $V_{G}=+30 \mathrm{~V}$ (red circles) and $V_{G}=0 \mathrm{~V}$ (blue squares). The line is meant to guide the eye to a $\mathrm{T}^{-3}$ dependence, characteristic of electron-phonon scattering. (b) Electron phase coherence length versus temperature plotted for $V_{G}=+30 \mathrm{~V}$ (red circles) and $V_{G}=0 \mathrm{~V}$ (blue squares). The line is meant to guide the eye to a $\mathrm{T}^{-3 / 4}$ dependence, which has also been observed in $\mathrm{ZnO}$ thin films.

scattering, reaches a peak, and then decreases to the lowest temperature we measured, 1.9 $\mathrm{K}$, where we report a mobility of $31 \pm 10 \frac{\mathrm{cm}^{2}}{V s}$ at $0 \mathrm{~V}$ gate bias and $92 \pm 2 \frac{\mathrm{cm}^{2}}{V s}$ at $+30 \mathrm{~V}$ gate bias. The effect of carrier concentration on mobility is significantly increased at lower temperatures; this may be the result of increased electron screening which reduces the effect of charged impurity scattering. ${ }^{52}$ Similar behavior is also reported for $\mathrm{ZnO}$ thin-films in the literature, with a mobility peak occurring near $110 \mathrm{~K} .^{53}$ The temperature dependence of mobility appears to scale more slowly than the $\mathrm{T}^{3 / 2}$ expected for impurity scattering in $\mathrm{ZnO}$ 

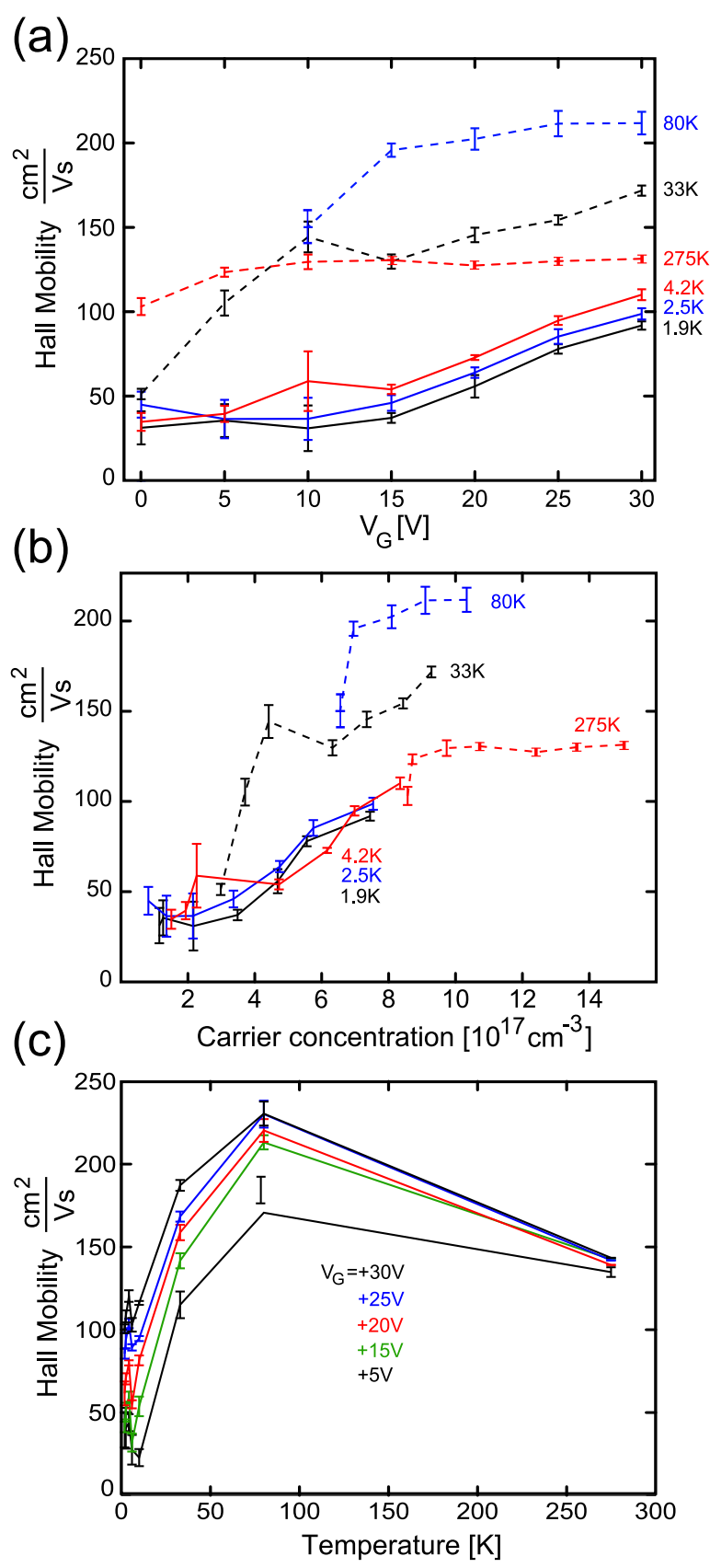

FIG. 5: (Color online) (a) Hall mobility versus gate bias for temperatures ranging from $1.9 \mathrm{~K}$ to 275 K. Mobility exhibits a larger dependence on carrier concentration at low temperature, where higher carrier density increases mobility. (b) Hall mobility versus carrier concentration. Mobility increases roughly linearly with carrier concentration and appears to saturate at higher carrier densities. (c) Hall mobility versus temperature. Mobility increases with temperature, reaches a maximum, and then decreases, consistent with previous reports on $\mathrm{ZnO}$ thin films. 
bulk crystals at low temperature. ${ }^{54-56}$ This scaling difference may be the result of a few factors, including weak localization suppressing mobility, a significant dependence on carrier density that affects impurity screening, or mobile, charged impurities which are described in Section VI. Although further investigation is needed to conclusively understand this temperature dependence, we suspect a significant contribution from the mobile impurities, as measurements were very sensitive to thermal cycling.

Nanoplates provide a useful platform to compare Hall mobility with the typically reported field-effect and effective mobilities in nanowires, ${ }^{21,34}$ because our 4 -wire contact geometry allows us to measure all three mobilities on the same nanoplate device. The more commonly reported field-effect mobility is calculated with a 2-terminal transconductance measurement according to: ${ }^{31}$

$$
\mu_{F E}=\frac{L}{W} \frac{g_{m}}{C_{o x} V_{D S}}
$$

where $L / W$ is the ratio of channel length to channel width; $g_{m}$ is the transconductance; $C_{o x}$ is the oxide capacitance per unit area; and $V_{D S}$ is the drain-source bias. In our device, we geometrically measure $L / W=2.71$ and $C_{o x}$ is calculated for a $300 \mathrm{~nm}$ thick $\mathrm{SiO}_{2}$ layer to be $11500 \mathrm{pF} / \mathrm{cm}^{2}$. We experimentally determine transconductance by applying a small D.C. source-drain bias, $V_{D S}$, across contacts $\mathrm{C} 2$ and $\mathrm{C} 4$ and measuring current while varying gate bias.

The effective mobility, as with the field-effect mobility, is determined using only a 2terminal measurement. Instead of estimating the mobility from the transconductance of the device, the source-drain conductance is used: ${ }^{31}$

$$
\mu_{E F F}=\frac{L}{W} \frac{g_{d}}{C_{o x}\left(V_{G}-V_{T}\right)},
$$

where $L / W$ is the ratio of channel length to channel width; $g_{d}$ is the drain conductance; $C_{o x}$ is the oxide capacitance per unit area; $V_{G}$ is the gate bias; and $V_{T}$ is the turn-on threshold.

The preference for Hall mobility is clear because it has no dependence on sample geometry. Additionally, Hall mobility is able to be determined independently from the carrier concentration. In contrast, field-effect mobility is limited to the gate bias at which the region of linear transconductance occurs. Effective mobility has the added complication that it also depends on the threshold voltage, which we show to be highly influenced by mobile impurities in the next section. 
Comparing the Hall mobility to field-effect mobility for the temperatures and carrier concentrations measured here, we conclude that the field-effect approximation tends to overestimate Hall mobility by about a factor of 2 in our devices. Field-effect mobility does report the correct order-of-magnitude, so it can be a useful approximation when a precise value is not necessary. Additionally, field-effect mobility provides no indication of the temperaturedependent mobility peak shown in Figure $5 \mathrm{c}$ and reported in the literature. We believe this to be an inherent limitation of the field-effect measurement, since it cannot determine mobility independent of carrier concentration.

\section{THRESHOLD BEHAVIOR}

During transconductance measurements at high temperatures (100 K and above), hysteresis was observed between successive sweeps, as shown in Figure 6a. This hysteresis is well-documented in the literature, with both surface adsorption ${ }^{34,57}$ and mobile impuri$\operatorname{ties}^{58,59}$ cited as possible mechanisms. Additionally, we observed that the threshold voltage can be varied over tens of volts by holding the gate at high or low bias for a period of time, as shown in Figure 6b. We note that the threshold voltage always moves toward the gate bias; that is, for $V_{G}=+30 \mathrm{~V}$ the threshold would move towards $+30 \mathrm{~V}$, and for $V_{G}=-30 \mathrm{~V}$ the threshold would move towards $-30 \mathrm{~V}$.

Our experimental data suggest that this hysteresis is the result of mobile Zn interstitials within the $\mathrm{ZnO}$ nanoplate. We believe this for several reasons: (1) the literature reports that $\mathrm{Zn}$ interstitials become mobile between $90-130 \mathrm{~K},{ }^{59}$ and we observed the hysteresis effect only for temperatures above $100 \mathrm{~K}$; (2) the activation energy that we measured in Section III corresponds well with what is expected for Zn interstitials; (3) for positive gate bias, the threshold moves to higher positive bias, which is consistent with positively-charged Zn interstitials in an n-type semiconductor; and (4) this effect is observed in a helium environment where we do not expect any oxygen to be available for adsorption, especially since we are able to repeatedly cycle the effect. Presumably, the $\mathrm{Zn}$ interstitials remain within the physical $\mathrm{ZnO}$ nanoplate, although more research is needed to determine the location of the active conduction channel and how it is affected by the Zn interstitials. We note that alkali metal ions could be introduced during typical semiconductor processing; however, we were careful to select chemical developers (MIBK and acetone) that do not 

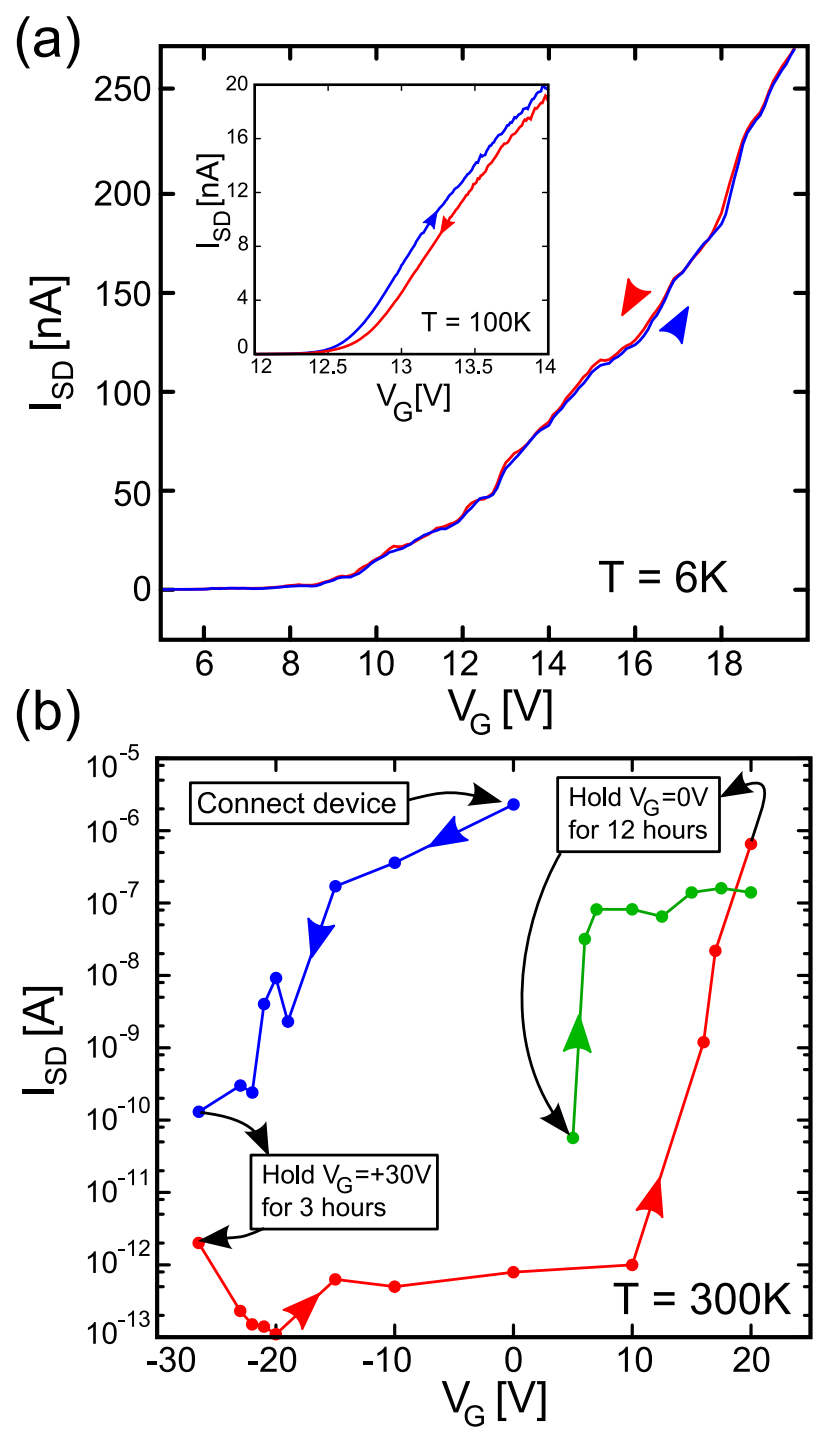

FIG. 6: (Color online) (a) Successive transconductance sweeps at $6 \mathrm{~K}$, showing no hysteresis. This is contrasted by (Inset) successive transconductance sweeps at $100 \mathrm{~K}$ for which hysteresis is evident. The effect is frozen out at temperatures below $100 \mathrm{~K}$. (b) A sequence of transconductance sweeps at $300 \mathrm{~K}$ taken after holding $V_{G}$ at different values. The threshold shifts by more than 30 $\mathrm{V}$ when $V_{G}$ is held at $+30 \mathrm{~V}$ for 3 hours. We believe this is caused by mobile, positively-charged Zn interstitials, as described in the text.

contain any metal ions. Additionally, EDS measurements do not show any evidence of alkali metals.

The fact that threshold voltage can be tuned over tens of volts may be of interest to device applications, as this behavior is both reversible and non-volatile. However, as discussed 
earlier, this variable threshold also makes accurate determination of effective mobility to be difficult, since $V_{T}$ in Eq. (4) is constantly changing.

\section{CONCLUSION}

In this paper, we present a comprehensive investigation into the electronic and magnetotransport properties of $\mathrm{ZnO}$ nanoplates grown by the vapor-liquid-solid (VLS) method. Unlike conventional nanostructures such as nanowires, the unique geometry of nanoplates allows us to conduct 4-terminal van der Pauw and Hall effect measurements. Using these techniques, we measure resistivity versus temperature and observe an activation energy (32 meV) characteristic of $\mathrm{Zn}$ interstitials that function as n-type donors. We report on magnetoresistance measurements showing weak localization in $\mathrm{ZnO}$ nanostructures and find the effect to be strongly dependent on temperature and only weakly dependent on carrier concentration. We obtain electron inelastic scattering lifetimes by fitting magnetoresistance data to three-dimensional weak localization theory. We observe that the phase coherence

lifetime scales as $\mathrm{T}^{-3}$, characteristic of electron-phonon scattering processes, and that the phase coherence length scales as $\mathrm{T}^{-3 / 4}$ and is 50-100 $\mathrm{nm}$ at $1.9 \mathrm{~K}$. We do not observe any evidence of anti-localization, suggesting that spin-orbit scattering is comparably weak and thus $\mathrm{ZnO}$ nanostructures may be useful for spintronic applications. Finally, we report on Hall mobility versus temperature and carrier concentration, finding a mobility of $\sim 100 \frac{\mathrm{cm}^{2}}{V s}$ at $275 \mathrm{~K}$, which is comparable to what is observed in $\mathrm{ZnO}$ thin films. We compare our calculated Hall mobility to the commonly reported field-effect mobility for nanowires, and find that the field-effect mobility overestimates the Hall mobility by a factor of 2 in our devices, but does function as a reasonable approximation. We comment on temperaturedependent hysteresis observed during transconductance measurements and attribute it to mobile, positively-charged Zn interstitial impurities.

\section{Acknowledgments}

This work was supported by NSF/NNIN through the use of their facilities at Harvard University's Center for Nanoscale Systems (CNS). The authors thank Warren Moberlychan and David Bell for transmission electron microscopy work. E.M.L. acknowledges a U.S. 
Department of Homeland Security Graduate Research Fellowship.

* Electronic address: likovich@post.harvard.edu

1 X. D. Wang, C. J. Summers, and Z. L. Wang, Nano Lett. 4, 423 (2004).

2 Y. C. Kong, D. P. Yu, B. Zhang, W. Fang, and S. Q. Feng, Appl. Phys. Lett. 78, 407 (2001).

3 P. D. Yang, H. Q. Yan, S. Mao, R. Russo, J. Johnson, R. Saykally, N. Morris, J. Pham, R. R. He, and H. J. Choi, Adv. Func. Mat. 12, 323 (2002).

4 D. C. Look, D. C. Reynolds, C. W. Litton, R. L. Jones, D. B. Eason, and G. Cantwell, Appl. Phys. Lett. 81, 1830 (2002).

5 K.-K. Kim, H.-S. Kim, D.-K. Hwang, J.-H. Lim, and S.-J. Park, Appl. Phys. Lett. 83, 63 (2003).

6 B. Xiang, P. Wang, X. Zhang, S. A. Dayeh, D. P. R. Aplin, C. Soci, D. Yu, and D. Wang, Nano Lett. 7, 323 (2007).

7 Q. Wan, Q. H. Li, Y. J. Chen, T. H. Wang, X. L. He, J. P. Li, and C. L. Lin, Appl. Phys. Lett. 84, $3654(2004)$.

8 K. Ueda, H. Tabata, and T. Kawaib, Appl. Phys. Lett. 79, 988 (2001).

9 L. Q. Liu, B. Xiang, X. Z. Zhang, Y. Zhang, and D. P. Yu, Appl. Phys. Lett. 88, 063104 (2006).

10 J. B. Cui and U. J. Gibson, Appl. Phys. Lett. 87, 133108 (2005).

11 M. H. Huang, S. Mao, H. Feick, H. Q. Yan, Y. Y. Wu, H. Kind, E. Weber, R. Russo, and P. D. Yang, Science 292, 1897 (2001).

12 M. Zimmler, J. Bao, F. Capasso, S. Muller, and C. Ronning, Appl. Phys. Lett. 93, 051101 (2008).

13 U. Ozgur, Y. I. Alivov, C. Liu, A. Teke, M. A. Reshchikov, S. Dogan, V. Avrutin, S.-J. Cho, and H. Morkoc, J. Appl. Phys. 98, 041301 (2005).

14 S. A. Wolf, D. D. Awschalom, R. A. Buhrman, J. M. Daughton, S. von Molnar, M. L. Roukes, A. Y. Chtchelkanova, and D. M. Treger, 294, 1488 (2001).

15 I. Appelbaum, B. Huang, and D. J. Monsma, Nature 447, 295 (2007).

16 Y. Ohno, D. K. Young, B. Beschoten, F. Matsukura, H. Ohno, and D. D. Awschalom, Nature 402, 790 (1999).

17 K. Masuko, A. Ashida, T. Yoshimura, and N. Fujimura, J. Appl. Phys. 103, 07D124 (2008).

18 H. Yu, J. Li, R. A. Loomis, L.-W. Wang, and W. E. Buhro, Nature Mat. 2, 517 (2003). 
19 Z. Fan, D. Wang, P.-C. Chang, W.-Y. Tseng, and J. G. Lu, Appl. Phys. Lett. 85, 5923 (2004).

20 Z. Fan, P.-C. Chang, J. G. Lu, E. C. Walter, R. M. Penner, C.-H. Lin, and H. P. Lee, Appl. Phys. Lett. 85, 6128 (2004).

21 Y. W. Heo, L. C. Tien, Y. Kwon, D. P. Norton, S. J. Pearton, B. S. Kang, and F. Ren, Appl. Phys. Lett. 85, 2274 (2004).

22 P.-C. Chang, Z. Fan, C.-J. Chien, D. Stichtenoth, C. Ronning, and J. G. Lu, Appl. Phys. Lett. 89, 133113 (2006).

23 J. Yoon, A. M. Girgis, I. Shalish, L. R. Ram-Mohan, and V. Narayanamurti, Appl. Phys. Lett. 94, $142102(2009)$.

24 R. S. Wagner and W. C. Ellis, Appl. Phys. Lett. 4, 89 (1964).

25 P. A. Hu, Y. Q. Liu, L. Fu, X. B. Wang, and D. B. Zhu, Appl. Phys. A 80, 35 (2005).

26 B. Illy, B. A. Shollock, J. L. MacManus-Driscoll, and M. P. Ryan, Nanotechnology 16, 320 (2005).

27 E. W. Petersen, E. M. Likovich, K. J. Russell, and V. Narayanamurti, Nanotechnology 20, 405603 (2009).

28 M. A. Zimmler, T. Voss, C. Ronning, and F. Capasso, Appl. Phys. Lett. 94, 241120 (2009).

29 Z.-M. Liao, K.-J. Liu, J.-M. Zhang, J. Xu, and D.-P. Yu, Phys. Lett. A 367, 207 (2007).

30 L. J. van der Pauw, Philips Tech. Rev. 20, 220 (1958).

31 D. K. Schroder, Semiconductor Material and Device Characterization (Wiley-Interscience, 2006), 3rd ed.

32 H. J. Fritzsche, J. Phys. Chem. Solids 6, 69 (1958).

33 X. D. Liu, E. Y. Jiang, and Z. Q. Li, J. Appl. Phys. 102, 073708 (2007).

34 J. Goldberger, D. J. Sirbuly, M. Law, and P. Yang, J. Phys. Chem. B 109, 9 (2005).

35 D. C. Look, J. W. Hemsky, and J. R. Sizelove, Phys. Rev. Lett. 82, 2552 (1999).

36 G. Bergmann, Phys. Rep. 107, 1 (1984).

37 P. A. Lee and T. V. Ramakrishnan, Rev. Modern Phys. 57, 287 (1985).

38 D. J. Bishop, R. C. Dynes, and D. C. Tsui, Phys. Rev. B 26, 773 (1982).

39 T. Andrearczyk, J. Jaroszynski, G. Grabecki, T. Dietl, T. Fukumura, and M. Kawasaki, Phys. Stat. Sol C 3, 1030 (2006).

40 X. D. Liu, E. Y. Jiang, and Z. Q. Li, J. Appl. Phys. 102, 073708 (2007).

41 Q. Xu, L. Hartmann, H. Schmidt, H. Hochmuth, M. Lorenz, R. Schmidt-Grund, D. Spemann, 
A. Rahm, and M. Grundmann, Thin Solid Films 515, 2549 (2006).

42 S. Hikami, A. I. Larkin, and Y. Nagaoka, Prog. Theor. Phys. 63, 707 (1980).

43 A. Kawabata, Solid State Commun. 34, 431 (1980).

44 A. Kawabata, J. Phys. Soc. Japan 49, 628 (1980).

45 R. G. Mani, L. Ghenim, and J. B. Choi, Solid State Commun. 79, 693 (1991).

46 X. Z. Yu, Y. Yang, W. Pan, and W. Z. Shen, Appl. Phys. Lett. 92, 092106 (2008).

47 F. A. Lindholm and R. W. Ayres, Proc. of the IEEE 56, 371 (1968).

48 S. M. Sze and K. K. Ng, Physics of Semiconductor Devices (Wiley-Interscience, 2007), 3rd ed.

49 E. M. Kaidashev, M. Lorenz, H. von Wenckstern, A. Rahm, H. C. Semmelhack, K. H. Han, G. Benndorf, C. Bundesmann, H. Hochmuth, and M. Grundmann, Appl. Phys. Lett. 82, 3901 (2003).

50 H. Kato, M. Sano, K. Miyamoto, and T. Yao, Jpn. J. Appl. Phys., Part 1 42, 2241 (2003).

51 K. Iwata, P. Fons, S. Niki, A. Yamada, K. Matsubara, K. Nakahara, and H. Takasu, Phys. Status Solidi A 180, 287 (2000).

52 N. W. Ashcroft and N. D. Mermin, Solid State Physics (Thomson Learning, 1976).

53 T. Edahiro, N. Fujimura, and T. Ito, J. Appl. Phys. 93, 7673 (2003).

54 A. R. Hutson, J. Phys. Chem. Solids 8, 467 (1959).

55 P. Wagner and R. Helbig, J. Phys. Chem. Solids 35, 327 (1974).

56 D. C. Look, D. C. Reynolds, J. R. Sizelove, R. L. Jones, C. W. Litton, G. Cantwell, and W. C. Harsch, Solid State Comm. 105, 399 (1998).

57 I. V. Markevich, V. I. Kushnirenko, L. V. Borkovska, and B. M. Bulakh, Solid State Comm. 136, 475 (2005).

58 B. T. Adekore, R. F. Davis, and D. W. Barlage, J. Appl. Phys. 101, 024902 (2007).

59 P. Erhart and K. Albe, Appl. Phys. Lett. 88, 201918 (2006). 